\title{
In-situ Atomic-Resolution Study of Stress-Induced Unusual Large Strain Plasticity of Semiconductor Nanowires
}

\author{
Z. Zhang and X. D. Han
}

Institute of Microstructure and Properties of Advanced Materials, Beijing University of Technology, 100022, Beijing, China

Employing tensile and/or bending stress via a newly developed in-situ atomic-lattice resolution electron microscopy method, we have, for the first time, successfully observed the brittle materials $\mathrm{SiC}$ and $\mathrm{Si}$ even $\mathrm{Si}_{\mathrm{x}} \mathrm{O}_{1-\mathrm{x}}$-nanowires (NWs) become highly ductile at room temperature. A crystalline structural evolution corresponding to the occurrence of unusual high ductility of the brittle materials includes the dislocation initiation, dislocation accumulation and the amorphous phase transition, finally the extended nano amorphous ductility processes at the necking region of the one dimensional nanowires. A corresponding deformation mechanism for these one dimensional nanowires under an external tensile force has been proposed based on MD simulations and it agrees well with our experimental results.

Amorphous solid materials as silicate glass or metallic glasses are usually with high hardness but brittleness in nature. The deformation mechanism of amorphous materials has two modes: at high stress and low temperature, the plastic deformation in metallic glass is inhomogeneous: the strain is concentrated in a few thin shear bands, while for silicate glass the plastic deformation is even much smaller before brittle failure. At lower stress and higher temperature, the deformation is homogeneous, which is atomic diffusion process for both types of glasses. The homogeneous flow regime includes liquid flow, which occurs above or near the glassy transition temperature, $T_{\mathrm{g}}$. Above $T_{g}$ superplasticity will occur for glasses with the nature of visco-plasticity.

We have reported, for the first time, a new phenomenon of large tensile plasticity of semiconductor nanowire through a phase transition of single crystal to amorphous under an external stress. These unusual mechanical properties of one-dimensional semiconductor nano materials with covalence bonds are fundamental important for understanding the nano-scale fracture and stress-induced band structure variation for high temperature semiconductors.

Reference

[1] X.D. Han, Z. Zhang and Z.L. Wang, "Experimental nanomechanics of one-dimensional nanomaterials by in situ microscopy" Nano, 2, 249 (2007)

[2] X.D. Han, Y.F. Zhang, K. Zheng, X.N. Zhang, and Z. Zhang, Y. J. Hao and X.Y. Guo, J. Yuan and Z. L. Wang, "Low-temperature in situ large strain plasticity of ceramic SiC nanowires and its atomic-scale mechanism" Nano Letters, 7, 452 (2007).

[3] X. D. Han, K. Zheng, Y. F. Zhang, X. N. Zhang, Z. Zhang, and Z. L. Wang "'Low-temperature in situ large-strain plasticity of silicon nanowires" Adv. Mater., 19, 2112 (2007).

[4] Zhang, YF; Han, XD; Zheng, K; et al., "Direct observation of super-plasticity of beta-SiC nanowires at low temperature", Adv. Func. Mater., 17, 3435 (2007)

[5] X.D Han et al., Lattice bending, disordering, and amorphization induced plastic deformation in a SiC nanowire, J. Appl. Phys., 98, 124307 (2005) 

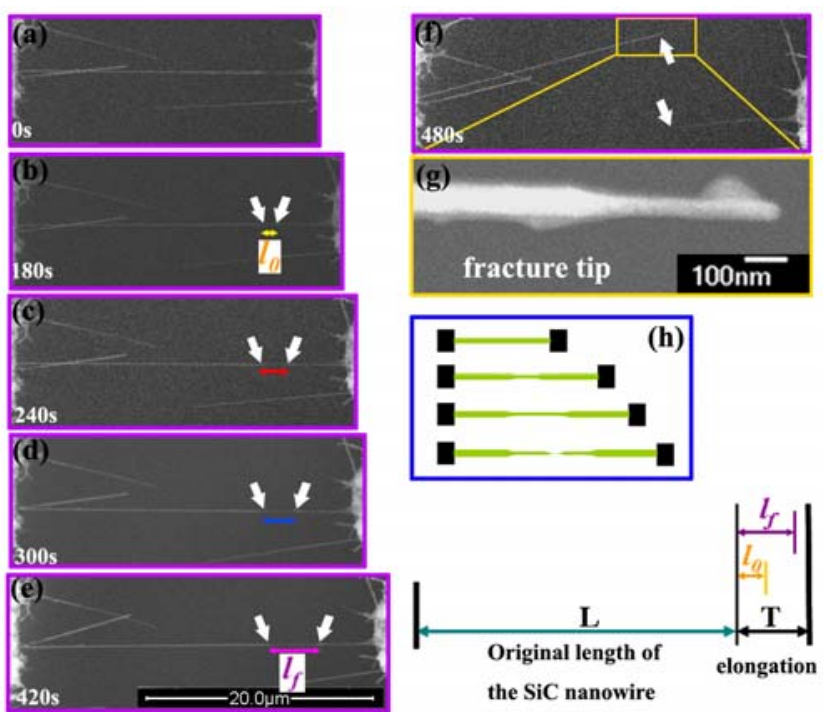

Figure 2 Direct atomic scale In-situ study of the Si NW large strain plasticity at room temperature. (a) and (b) are the TEM images captured at the two ends of the tensile experiments: initial of the tensile experiment (a) and the fracture of the NW (b). (c) and (d) are the atomic scale images of the tensile $\mathrm{Si}$ NW. (c) is the Si NW with a character initial elastic-plastic transition. The regions with emerging dislocations are indicated by black-dashed lines. A thin amorphous layer is indicated by a pair of blue arrows. (d) shows the NW with large strain plasticity and a surprising necking feature indicated by character B. The A and $\mathrm{C}$ zones in (d) are with high density dislocations partially amorphization.

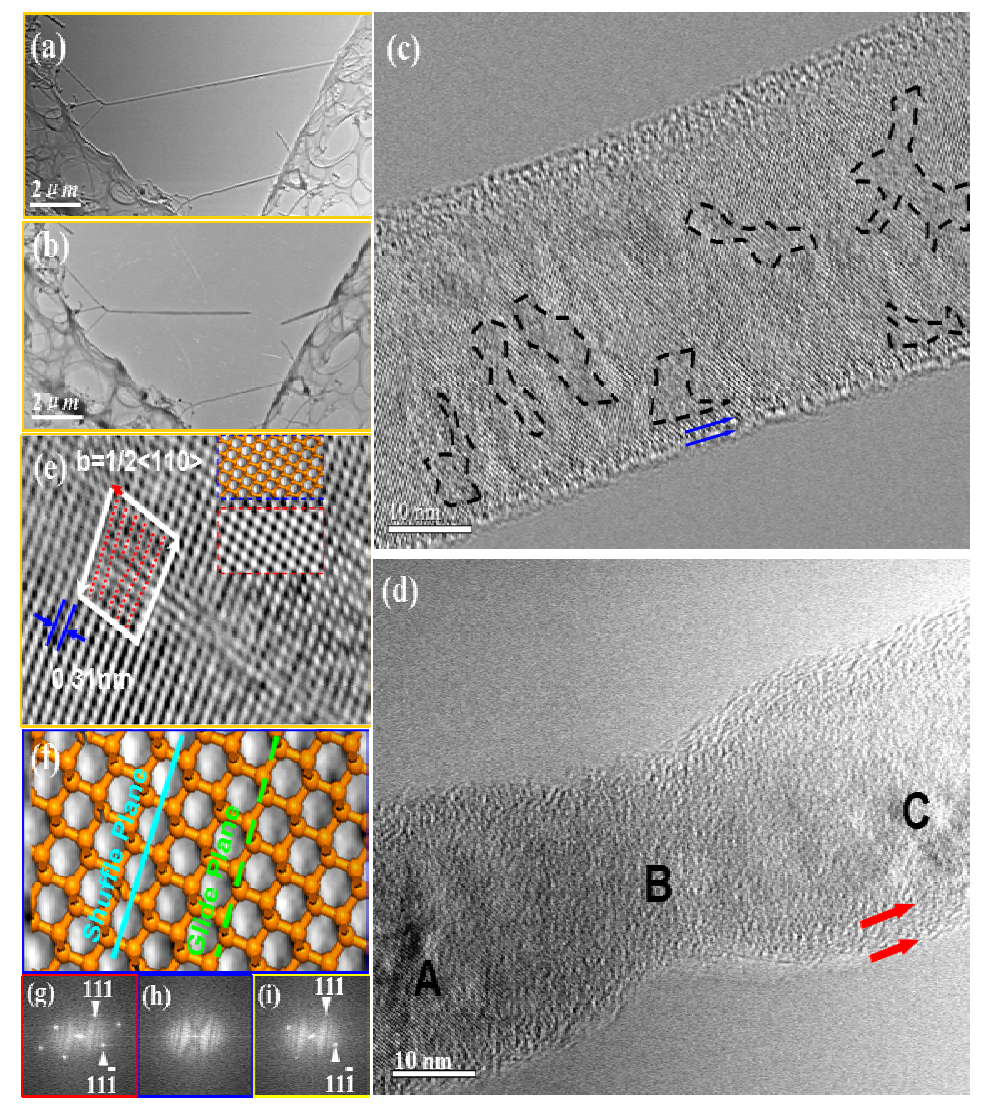

The surface amorphous layer expands to be thicker as indicated by the red arrow pair in (d). An example of dislocation is shown in (e), the Burgus vector is determined by the figure as $1 / 2$ [110]. (f) illustrates the (111) shuffle and glide planes and we suggest that the dislocation activity occurred at the shuffle (111) plane. (g) through (i) are the fast Fourier transformation patterns corresponding to A, B and C zones of (d). 